\title{
Ain- \\ Los posibles beneficios de la conciliación
}

\author{
Salvatore Moccia \\ PROFESOR COLABORADOR DOCTOR \\ UNIVERSIDAD CEU - CARDENAL HERRERA \\ salvatore.moccia@uch.ceu.es
}

Resumen: A principios de este nuevo siglo, uno de los retos más importantes de las sociedades económicamente más avanzadas es la conciliación de la vida laboral con la familia.

De hecho, los enormes cambios socio-demográfico-económicos de los últimos años han comportado la necesidad de reorganizar la estructura de las relaciones trabajo-familia. La integración de la conciliación de la vida laboral y familiar en la cultura empresarial responde no sólo a razones de carácter legal, sino sobre todo a razones de carácter organizativo/empresarial, presentándose como una seria y potente inversión en el capital humano. El objetivo de este trabajo es reflexionar sobre los posibles beneficios, tanto para las empresas como para las personas, de la introducción de medidas de conciliación en el ámbito de la cultura empresarial, ya que con las herramientas del management tradicional sólo se puede forzar a los individuos a ser obedientes y diligentes, pero no se 
puede conseguir que sean creativos, comprometidos, ilusionados y, sobre todo, leales y fieles al proyecto empresarial. Por tanto, la conciliación trabajo-familia podría representar el necesario esfuerzo hacía una tipología de humanismo empresarial en el cual la persona -en su totalidad-se transforme en elemento central y crucial de la actividad económica.

Palabras clave: Conciliación, Relación trabajo y familia, Felicidad y bienestar, Beneficios para las empresas, Beneficios para los trabajadores.

\section{The potential benefits of reconciliation}

Abstract: At the turn of the new century, one of the most important challenges in more economically advanced societies is reconciling work and family life.

In fact, the enormous socioeconomic and demographic changes of recent years have led to a need to reorganise the structure of workfamily relationships. Integrating reconciliation of an individual's working and family life in business culture responds not only to reasons of a legal nature, but above all to reasons of an organisational/entrepreneurial nature, and is held to be a serious and powerful investment in human capital. The aim of this study is to reflect on the potential benefits, both for businesses and individuals, of introducing reconciliation measures where corporate culture is concerned, since traditional management tools do nothing more than force individuals to be obedient and diligent, without getting them to be creative, committed, excited and above all, loyal and faithful to the business project. Therefore, reconciling work and family might represent the necessary effort towards achieving a type of corporate humanitarianism in which the individual is fully transformed into the crucial and central focus of economic activity.

Keywords: Reconciliation, Work-Family Relationships, Welfare and Happiness, Benefits for businesses; Benefits for workers. 


\section{Los posibles beneficios Ide la conciliación}

Recibido: 31-05-2011

Aceptado: 28-11-2011

Salvatore

Moccia

\section{Introducción}

En la actualidad asistimos a un nuevo tipo de sociedad, denominada Post-Capitalista (Drucker, 1993), Post-Industrial (Bell, 1973), sociedad de consumo o sociedad de la información y el conocimiento (Castells, 1999). Mientras que durante los siglos precedentes el motor de la economía y, en buena parte, la definición de la sociedad, se regían por valores agrícolas y materiales, y la necesidad y la supervivencia eran algunas de las preocupaciones clave para los individuos, hoy, como fruto de las grandes transformaciones acaecidas durante el siglo XX, los valores imperantes parecen relacionarse en gran medida con el bienestar, la calidad de vida y el consumo (Quintanilla, 2008), desplazando la atención desde la necesidad a la apetencia.

A principios de este nuevo siglo, uno de los retos más importantes de las sociedades económicamente más avanzadas es la conciliación de la vida laboral con la familia.

De hecho, los enormes cambios socio-demográfico-económicos de los últimos años han comportado la necesidad de reorganizar la estructura de las relaciones trabajo-familia. La integración de la conciliación de la vida laboral y familiar en la cultura empresarial responde no sólo a razones de carácter legal, sino sobre todo a razones de carácter organizativo/empresarial, presentándose como una seria y potente inversión en el capital humano. Como señalan Olcese, Rodriguez y Alfaro (2008), "diversos hechos, entre los que cabe destacar la globalización y la revolución de las tecnologías de la información y comunicación, han ocasionado que la economia (y la sociedad) haya pasado de estar basada en los recursos naturales a fundamentarse en el conocimiento". En la actualidad, las personas son consideradas la fuente más importante de ventaja competi- 
tiva de las empresas y, por tanto, su gestión debe dejar de percibirse no como un coste, sino como una inversión de carácter estratégico. La clave de la economía moderna es, precisamente, el énfasis teórico y práctico en la fertilidad de recursos. Ya Adam Smith, que pasa por ser el padre del capitalismo doctrinal, advirtió que la riqueza de las naciones no consiste -como pretendía el mercantilismo- en el territorio sobre el que los Estados ejercen su soberanía, ni en el conjunto de bienes naturales y culturales que esas tierras atesoran. El gran hallazgo de Adam Smith consiste en haber descubierto que la riqueza de las naciones estriba en la creatividad de sus ciudadanos, en su capacidad de acometer proyectos que deparen un beneficio económico para los individuos (Llano, 1997).

El objetivo de este trabajo es reflexionar sobre los posibles beneficios, tanto para las empresas como para las personas, de la introducción de medidas de conciliación en el ámbito de la cultura empresarial, ya que con las herramientas del management tradicional sólo se puede forzar a los individuos a ser obedientes y diligentes, pero no se puede conseguir que sean creativos, comprometidos, ilusionados y, sobre todo, leales y fieles al proyecto empresarial. Por tanto, la conciliación trabajo-familia podría representar el necesario esfuerzo hacía una tipología de humanismo empresarial en el cual la persona -en su totalidadse transforme en elemento central y crucial de la actividad económica.

\section{Los cambios socio-demográfico-económicos}

Los cambios socio-demográfico-económicos ocurridos en los últimos años se han producido básicamente por lo siguiente:

- globalización de las relaciones económicas y comerciales, lo que, a su vez, ha determinado un reajuste de la estructura organizativa y de las maneras de organizar el trabajo;

- progresivo abandono de la agricultura y aumento del peso de los sectores industriales y servicios con la consecuente despoblación de las áreas rurales y el aumento de la población en las ciudades;

- incremento continuo de la actividad económica, que ha determinado un periodo de expansión en el que se ha pro- 
ducido un aumento importante de la ocupación de ambos géneros;

- abandono del modelo de familia tradicional y creación de núcleos familiares aislados geográficamente del contexto familiar originario;

- progresivo aumento del nivel de escolaridad;

- progresiva reducción/modificación de la oferta formativa individual, y adopción de modelos formativos más estandardizados y menos aptos para la resolución de los problemas individuales.

Estos cambios han determinado profundas modificaciones en la realidad cotidiana familiar. En particular, si es verdad que las mujeres siguen siendo el pilar familiar en las tareas domesticas, a esto se le he añadido las siguientes necesidades:

- salir del núcleo familiar para incorporarse al mundo laboral con el fin de aportar dinero a la familia a causa de los incrementos de costes debidos a la creación de núcleos familiares aislados geográficamente del núcleo familiar original y concentrados en áreas geográficas (ciudades) en media más caras;

- cuidar de los hijos a causa de la ausencia de los abuelos u otros familiares del núcleo familiar;

- disponer de más tiempo para cubrir las distancias entre empresa y hogar;

- disponer de ayuda para acudir a las necesidades de personas de la tercera edad, eventualmente presentes en el núcleo familiar.

\section{La obligación de nuevas soluciones organizativas}

De todo este cuadro, surge para las empresas la obligación de encontrar nuevas soluciones organizativas que permitan conciliar el trabajo con las necesidades del núcleo familiar. Es imprescindible que las empresas pongan en marcha unos programas y unas prácticas de conciliación que permitan a las personas encontrar sentido en lo que hacen, sin ver mermada su productividad por unas preocupaciones que no provienen de la empresa sino de la familia. En consecuencia, es importante, a 
parte de las obligaciones legales, conciliar la vida laboral con la vida familiar a través de programas específicos que, invirtiendo en las personas que componen la empresa, puedan ayudar, al mismo tiempo, a mejorar la productividad laboral.

Esto implica un cambio de mentalidad a nivel estratégico ya que es preciso que no se vea la conciliación como una mala inversión a la que la empresa está obligada por la ley, sino como una oportunidad única de aumentar la productividad de la empresa a través de un renovado bienestar de las personas. Pero, para que se produzca este cambio tan profundo en la cultura empresarial $y$, sobre todo, para que esta nueva cultura impregne la empresa a todos los niveles y perdure en el tiempo, de modo que no reduzca a una moda pasajera, es imprescindible que el equipo directivo crea en esta nueva visión y, sobre todo, que apueste por ella y fomente el cambio. En particular, es necesario que el plan de conciliación indique con exactitud los beneficios tanto para las personas como para la empresa, de manera que sea más fácil su introducción.

\section{Los beneficios para las empresa y las personas}

Entre los posibles beneficios para las personas, podemos señalar los siguientes:

- La reducción del estrés y de la ansiedad laboral, y, naturalmente, el aumento del índice de productividad y la reducción del índice de baja y de rotación del personal;

- El aumento de la motivación, de la lealtad a la empresa y de la ilusión en el trabajo que se desarrolla;

- La posibilidad de un mayor desarrollo personal y profesional, ya que el trabajador, encontrándose a gusto en la empresa, se preocupará más de formarse como persona (buen compañero de trabajo) y como profesional (que conoce realmente todas las facetas de lo que hace);

- El aumento de la felicidad y de la estabilidad emocional, con mayor presencia de emociones positivas, frente de la reducción de las emociones negativas. Muchas investigaciones empíricas muestran que las medidas macro del bienestar del trabajador están relacionadas positivamente con el rendimiento. Por ejemplo, en una investigación lle- 
vada a cabo en Inglaterra en el año 2006, se ha demostrado que los trabajadores que trabajan con emociones positivas obtienen mejores resultados que los trabajadores con emociones negativas.

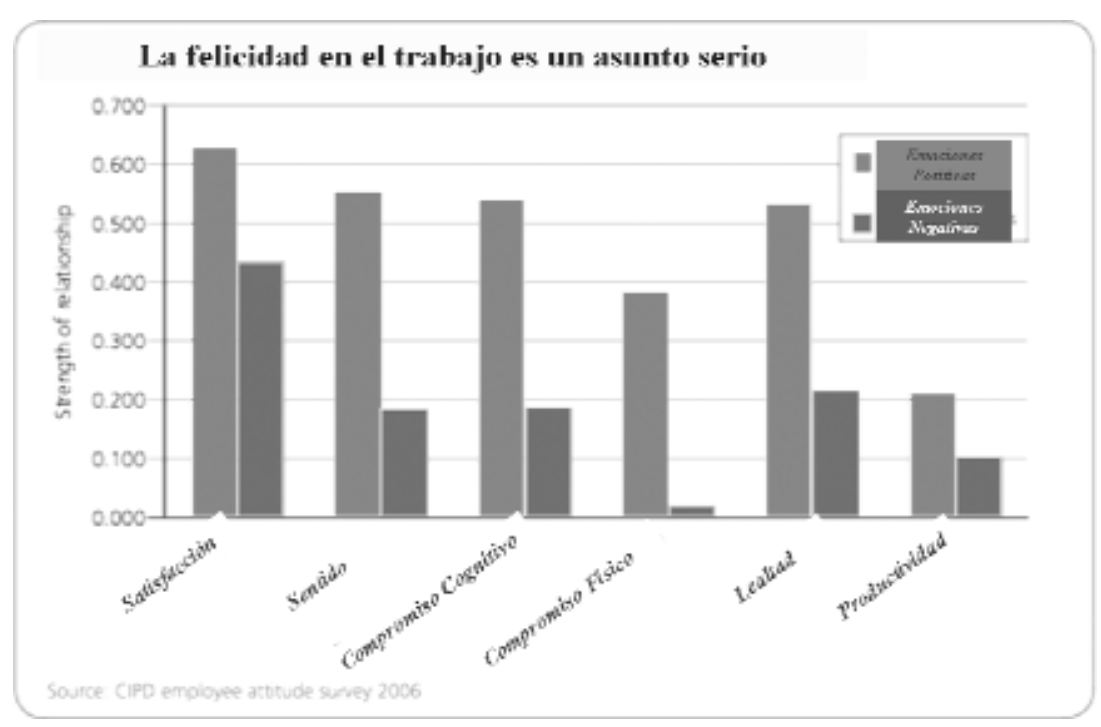

Fuente: Truss, K., et Al. (2006).

Entre los posibles beneficios para las empresas, se señalan los siguientes:

1) Mayor compromiso del personal con la empresa. La clave general para crear un mayor compromiso dentro de la empresa es el conjunto de acciones establecidas para que las personas trabajen más a gusto. Entre estas acciones, la conciliación es de vital importancia, ya que afecta directamente al nivel personal. Como seres humanos, todos queremos recibir un trato personal y detestamos que nos traten como si solo fuéramos parte de una masa. Nos gusta que la empresa escuche nuestros problemas, los entienda y, sobre todo, se comprometa en resolverlos. Si la empresa espera conseguir el compromiso de las personas, debe tratarlas como personas completas, con sus potencialidades y debilidades, e incluso con sus proble- 
mas de carácter familiar, que si no se resuelven, se transforman en catalizadores de unas energías que, en lugar de ser dirigidas al trabajo, se dirigirán inevitablemente hacia la familia. Por otro lado, si se consigue el compromiso de los trabajadores, las mismas empresas obtendrán importantes beneficios, incluso de carácter economíco. De hecho, son muchos los autores que, de una manera $u$ otra, confirman desde una perspectiva científica que el compromiso representa el motor que impulsa a las personas a alcanzar los objetivos individuales y de la organización. En 2005, la consultora Towers Perrin llevó a cabo un estudio sobre 86.000 empleados de grandes y medianas empresas de 16 países, con el fin de medir el compromiso medio de los trabajadores. Las conclusiones del estudio evidencian que «la inmensa mayoría de los empleados de todos los niveles de una empresa no están completamente comprometidos con su trabajo». Según este estudio, sólo un $14 \%$ de los empleados de todo el mundo están comprometidos con su trabajo, mientras que el $24 \%$ se siente totalmente desconectado. En pocas palabras, aproxidamamente el $85 \%$ de los trabajadores no dan de sí todo lo que podrían.

Milne (2007), confirmando que todas las personas exitosas tienen en común un mismo elemento -el compromiso con lo que hacen-, evidencia que «si los trabajadores se comprometen con lo que hacen, serán más felices y más productivos. Además, con sus actitudes positivas contagiarán a los clientes y a los demás trabajadores y no se irán de la empresa". Por su parte, Seo, Barret y Bartunek (2004) confirman la importancia de las emociones en la vida empresarial, pues estos investigadores tienden a considerar las emociones como una antítesis de la racionalidad fría, que actúa en contra de la gestión eficaz. Por eso -siguen diciendo los mismos autores-, el interés por el papel de las emociones en las prácticas empresariales es cada vez mayor en los investigadores de gestión empresarial, habiéndose creado ya cuatro corrientes de investigación. Además, los recientes avances empíricos y teóricos en el campo de la neurobiología y de la psicología han puesto de manifiesto 
que la emoción está relacionada con la motivación para el trabajo, y que todas las teorías de la motivación que no contemplen la emoción resultan incompletas.

2) Mejora del clima laboral, con consecuente mejora de las relaciones laborales y sindicales.

Se puede definir el clima laboral como el conjunto de características relativamente permanentes del ambiente laboral que influyen en el comportamiento de los empleados. El clima laboral difiere de la cultura de la organización por la presencia de las siguientes características peculiares:

a) está compuesto por una pluralidad de dimensiones;

b) depende de las percepciones individuales más que de las condiciones ambientales objetivas;

c) no es un fenómeno directamente visible, pero sus consecuencias -positivas y negativas- son muy tangibles en el comportamiento;

d) es relativamente estable, pero no inamovible.

Según las investigaciones empíricas, un clima laboral favorable aporta los siguientes beneficios a los trabajadores:

1. entusiasmo,

2. cohesión,

3. satisfacción,

4. creatividad,

5. amistad,

6. responsabilidad social,

7. reducción de las ansiedades y del estrés,

8. reducción del aburrimiento y,

9. aumento de la confianza.

3) Reducción de los índices de rotación del personal y reducción de las bajas por enfermedad.

La Organización Mundial de la Sanidad (OMS) estima que en el 2020 la depresión será la segunda causa de inhabilidad laboral, y que en Europa los costes de la

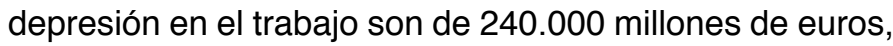
de los cuales 136.000 se deben a la perdida de productividad. La depresión laboral, a través del absentismo labo- 
ral y del presentismo (se produce cuando una persona está físicamente en su puesto de trabajo, pero no lo desempeña al $100 \%$ ), determina una reducción de la productividad colectiva, de la producción, de la calidad y de la reputación de la empresa. Además, la OMS señala que el número las personas que se quitan la vida cada año es habitualmente mayor que el de las muertes por enfrentamientos bélicos o por homicidios. Por ejemplo, en el año 2002, fueron 873.000 las personas que se quitaron la vida, frente a los 172.000 muertos por guerras o los 559.000 victimas de homicidios. Además, el problema adquiere una dimensión mayor si se tiene en cuenta que cada suicidio siempre está acompañado por 10-15 parasuicidios, o sea, personas que se autolesionaron sin llegar a morir.

4) Aumento de la productividad, creatividad e innovación.

Las personas son seres inteligentes que pueden contribuir activamente a la eficiacia de la organización. Este potencial, según McGregor (2006), «no sólo sirve para realizar un mayor esfuerzo, sino también para aplicar el ingenio, la creatividad para resolver problemas, la aceptación de la responsabilidad, el liderazgo, el desarrollo de habilidades del conocimiento y el discernimiento". Las personas son fuentes de ciertos conocimientos tácitos que la empresa no puede adquirir por contrato; son, además, fuentes de ventaja competitiva duradera. A medida que el mundo se vuelve más complejo, el modelo organizativo más apropiado el modelo humano, ya que la innovación no la hacen las maquinas sino las personas. De hecho, el economista Xavier Sala-i-Martín afirma que el $71 \%$ de las políticas innovadoras de las empresas procede de sus empleados, mientras que el $21 \%$ tiene su origen en las casualidades y, finalmente, solo el $8 \%$ procede del proceso formal de $\mathrm{I}+\mathrm{D}+\mathrm{i}$. Por tanto, si diseñamos las empresas con politicas de conciliación que permitan a las personas vivir bien, las personas serán más felices, estarán más dispuestas a abrirse para donar la creatividad que llevan dentro, serán más productivas y menos oportunistas. 
Según un reciente estudio publicado por Randstan (enero 2008), el entorno laboral tiene bastante o mucha influencia sobre la productividad de los empleados. EI $90 \%$ de los trabajadores considera que el lugar donde trabaja influye "bastante o mucho" en su productividad. El sondeo se ha llevado a cabo entre profesionales de recursos humanos mediante una encuesta 'online' y una serie de entrevistas telefónicas dirigidas a determinar si el grado de felicidad de los empleados influye en su productividad. Entre el $65 \%$ y el $75 \%$ de las personas encuestadas opinan que el medio físico en el que los futuros empleados tendrán que trabajar les influye a la hora de decidir unirse o no a la empresa. Y más del $90 \%$ opinan que el grado de implicación del trabajador depende del lugar de trabajo. Por su parte, el $92 \%$ de los profesionales de RR.HH. piensan que el entorno de trabajo tiene bastante o mucho impacto en el bienestar de los trabajadores en su empresa. Y hasta el $82 \%$ piensa igual respecto a la satisfacción en el trabajo.

5) Retención e incorporación del talento.

En un mundo donde los ciclos vitales de la economía se reducen drásticamente, la innovación es la única posibilidad de las compañias para sobrevivir. Normalmente se suele creer que la innovación es el resultado del trabajo de un reducido número de supertalentos a los que, primero, hay que incorporar y, luego, retener. Pero esta visión es parcialmente equivocada, ya que son muchos los investigadores en temas de gestión empresarial que creen que cada trabajador tiene su propio talento. El problema, por tanto, reside en cómo descubrir, organizar y retener el talento de cada uno de los trabajadores de la empresa. La creatividad es una capacidad humana; la auténtica barrera para retener los talentos reside en la capacidad de la organización de ser más humana, de tener en cuenta el aspecto humano de cada trabajador. A cambio, recibirá sus componentes intangibles, como la lealtad y la creatividad.

6) Mejora de las relaciones con los clientes, ya que unos trabajadores satisfechos conllevan unos clientes satisfechos. 
El deseo de felicidad es universal. La idea de que el trabajo es un obstáculo para la felicidad está bastante extendida. La causa de esta opinión tan generalizada es doble. Por una parte, todo trabajo exige esfuerzo, sacrificio y abnegación, y eso parece oponerse al ideal de felicidad como bienestar. Por otra, las condiciones en las que la persona tiene que realizar su trabajo no son, en muchas ocasiones, las más adecuadas para hacer de esa actividad una fuente de felicidad.

El hecho es que las personas que se sienten felices, están absortas en sus actividades y experimentan una enorme satisfacción en lo que hacen. En realidad, trabajan para ser felices, con ganas de realizarse y desarrollarse a nivel personal, y ponen siempre la satisfacción personal por encima del deseo de ganar dinero.

Según los investigadores en tema de gestión empresarial, hay diez razones que pueden justificar el hecho de que las personas felices en el trabajo sean elementos de empuje para mejorar los resultados empresariales. Son las siguientes:

- Trabajan mejor en equipo

- Son más creativas

- Solucionan los problemas en vez de quejarse de los problemas

- Tienen más energía

- Son más optimistas

- Están más motivadas

- Enferman menos

- Aprenden más rápidamente

- Se preocupan menos de los errores y, como consecuencia, fallan menos

- Toman mejores decisiones

De hecho, las empresas y, en particular, los departamentos de recursos humanos, están descubriendo una de las claves para mejorar la productividad empresarial: el trabajador feliz. Está comprobado que los empleados felices están más comprometidos con la empresa, con sus objetivos y con sus proyectos específicos, incrementando de este modo su rendimiento. 
Algunos autores afirman que existen claras evidencias de que las medidas macro del bienestar del trabajador están relacionadas positivamente con el rendimiento. Señalan además que las prácticas laborales diseñadas para incrementar el bienestar del trabajador proporcionan un retorno significativo de la inversión en términos de beneficio, y otros valiosos resultados empresariales.

Un estudio meta-analítico, el de Staw, Sutton y Pelled (1994), concluye que cuanto mayor sea la felicidad en el trabajo, más alta será la productividad. De hecho, el economista Zamagni afirma que «los economistas se equivocaron al escribir en sus libros que eficiencia y felicidad estaban reñidas, no es verdad en absoluto (...) Si las empresas hoy no son capaces de resolver el problema de la felicidad, no podrán ser competitivas". Uno de los gurús de la gestión de la calidad total, Hokuro Ishikawa, había afirmado ya que «una organización cuyos miembros no son felices y no pueden alcanzar la felicidad, no merece existir».

7) Mejoría de la imagen y de la credibilidad de la empresa.

La imagen de la empresa es parte del capital de la misma. La reputación de una empresa/marca se puede definir como la identificación del consumidor con la imagen de la empresa/marca cuando reconoce en esta unos valores funcionales, sociales y de autoexpresión de su personalidad que le proporcionan una experiencia positiva en relación con ella.

La reputación es un poderoso factor de diferenciación de productos y servicios, dado que la saturación de los mercados comerciales y la homogeneidad de la oferta han producido una gran igualdad.

Según unos expertos en el tema de la reputación empresarial, al final de la década de los años treinta el valor de una empresa en EEUU se correspondía en un $70 \%$ con sus activos tangibles. En los años noventa, ese porcentaje se había invertido, convirtiendo los activos intangibles en la fuente principal de valor de las empresas.

Hoy en día, gracias sobre todo a las presiones que se reciben por parte de grupos de consumidores muy fuer- 
tes, la imagen de la empresa resulta imprescindible para permanecer en un negocio. Ya son varios los productos que utilizan etiquetas en las cuales se apunta algo similar a esto: "Producto realizado sin la explotación de los trabajadores". Las medidas de conciliación sirven no solo para respectar las leyes, sino también para mejorar la imagen y la credibilidad de la empresa.

De hecho, ya son muchas las empresas que cada año participan al "Great place to work", "mejor lugar de trabajo", aportando novedades sobre todo en tema de conciliación vida-trabajo. Por ejemplo, en el año 2007, Microsoft España fue galardonada como el mejor lugar de trabajo (Best Work Places-España 2007) gracias a su proyecto de cambio cultural, nacido para mantener alta la satisfacción del empleado dentro de la Compañía. El programa -denominado 3 P's- incluía iniciativas en tres sectores: Profesional, Productividad, Personas. En particular, respecto a las iniciativas en favor de las personas, se introdujeron las siguientes medidas de conciliación: horario flexible, teletrabajo, protección de la situación de paternidad, ampliación del descanso por maternidad, ampliación por el período de lactancia, flexibilización del período de descanso maternal, jornada intensiva los viernes y durante el verano. Los resultados del proyecto han sido:

- Mejora en el equilibrio personal-profesional.

- Autoridad delegada e incremento de la responsabilidad.

- Beneficios y productividad.

- Satisfacción en familias y empleados.

- Retención mujeres/madres.

- Empleados más satisfechos, alegres = empresa más rentable y atractiva.

- Impacto en la marca.

- Reducción de cuadros médicos, stress, rotación.

- Orgullo de pertenencia.

- Desarrollo del potencial y de la creatividad, sensación de libertad.

- Reconocimiento en el mercado español y en Microsoft Europa. 


\section{Conclusiones}

La conciliación es un factor esencial para las organizaciones del siglo XXI y puede conllevar beneficios tanto para las empresas como para los trabajadores. Pero esta no debe dirigirse sólo a la mujer, ya que las dificultades de conciliar la vida laboral con el resto de las facetas de la vida no es una cuestión que afecte sólo a ella. Es, por tanto, indispensable, que la gestión empresarial actual entienda la conciliación no solamente como una herramientas más para controlar el comportamiento de las personas y para ajustarlas a las necesidades de la organización, sino como la posible generación de un entorno en el que la dirección, a través del establecimiento de un oportuno clima laboral, demuestre un sentido de aprecio y de respeto hacia los trabajadores/as. Las personas pueden y deben contribuir activamente a la eficiencia de la organización. Son fuentes de unos conocimientos tácitos que no podemos adquirir por contrato; son, además, fuentes de ventaja competitiva duradera. Conforme el mundo se vuelve más complejo, el modelo organizativo más apropiado es el modelo humano, ya que la innovación no la hacen las máquinas sino las personas. En definitiva, las empresas, y en particular los departamentos de recursos humanos, deben descubrir la potencialidad de la conciliación como medida para mejorar no solo la productividad laboral, sino incluso el compromiso del trabajador. Cuanto más se avance en este campo, más fácil será contar con ciudadanos satisfechos, participativos e involucrados en el devenir de la comunidad a la que pertenecen.

\section{Referencias}

ABRIL STOFFELS, R. (Coord.) (2009). Fundamentación teórica y comparativa de las políticas públicas de conciliación. Tirant, Valencia (versión en internet: http://www.bsocial.gva.es/portal/portal?id $=44971 \& \mathrm{sec}=1812011162523$ ).

ARAGÓN MEDINA, J., CÁNOVAS MONTERO, A. y ROCHA SÁNCHEZ, F. (2005). "Las políticas de conciliación de la vida laboral y familiar en las Comunidades Autónomas". Madrid. Cinca. 
ASHKANASAY, N. y DAUS, C.S. (2002). "Emotion in the workplace: the new challenge for managers". Academy of Management Executive, Vol. 16, n. 1, pp. 76-86.

AVOLIO, B.J.; HOWELL, J.M. y SOSIK J.J. (1999). "A funny thing happened on the way to the bottom line: humor as a moderator of leadership style effects", Academy of Management Journal, Vol. 42-2, pp. 219-227.

BAKER, D.; GREENBER, C. y HEMINGWAY, C. (2007). "Empresas felices = Empresas rentables", Gestión 2000, Barcelona.

BELL, D. (1973). "The coming of post industrial society". New York. Basic Books.

BOWEN, D.; LEDFORD, G. y NATHAN, B. (1991). "Hiring for the organization, not the job", Academy of Management Executive, Vol. 5, n. 4, pp. 35-51.

CARBELO, B. y JÁUREGUI, E. (2006). "Emociones Positivas: humor positivo", Papeles del Psicólogo, Vol. 27 (1), pp. 1830.

CARR, E. (2007). "The expanding vision of positive behavior support", Journal of positive behaviour interventions, Vol. 9, n. 1, pp. 3-14.

CASADO APARICIO, H. (2006). "Los desafíos de la conciliación de la vida familiar y profesional en el siglo XXl". Madrid. Biblioteca Nueva.

CASTELLS, M. (1999). "La era de la información: economía, sociedad y cultura: la sociedad Red". México D.F. Siglo XXI.

CHINCHILLA, N. (2007). "Dueños de nuestro destino: cómo conciliar la vida profesional, familiar y personal", Barcelona. Ariel.

CHINCHILLA, N. y LEON, C. (2004). "La ambición femenina: cómo re-conciliar trabajo y familia". Madrid. Taurus.

CORRALES INGLÉS, R. (2010). "La igualdad, un trabajo en equipo: la corresponsabilidad en el ámbito familiar". Sevilla. Instituto Andaluz de la Mujer.

CRUZADO, M. y VELASCO, A. (2005). “¿Vives o trabajas?: flexibilidad laboral y equilibrio personal generan rentabilidad $y$ satisfacción". Madrid. LID Editorial Empresarial.

DRUCKER, P. (1993). "The post capitalist society". New York. Harper Business.

DWYER, T. (1991). "Humor, power, and change in organizations", Human Relations, Vol. 44, n. 1, pp. 1-19. 
EREZ, A. y ISEN, A. (2002). "The influence of positive affect on the components of expectancy motivation", Journal of Applied Psychology, vol. 87, $\mathrm{n}^{\circ}$ 6, pp. 1055-1067.

FORD, R.; McLAUGHLIN, F. y NEWSTROOM, J. (2003). "Questions and answers about fun at work", Human Resource Planning, vol. 26, $\mathrm{n}^{\circ} 4$, pp. 18-33.

FREDRICKSON, B., L. (2003). "The value of positive emotions", American Scientist, Vol. 91, n. 4, pp. 330-337.

FREDRICKSON, B.L. y LOSADA, M.F. (2005). "Positive affect and the complex dynamics of human flourishing", American Psychologist, Vol. 60, n. 7, pp. 678-686.

GALA DURÁN, C. (2007). "La conciliación de la vida laboral y familiar del personal al servicio de las entidades locales". Barcelona. Diputación de Barcelona.

GARCÍA-MINÁ FREIRE, A. y CARRASCO GALÁN, M. (2005). "El ajuste trabajo-familia desde una perspectiva de género". Madrid. Universidad Comillas.

GAVÍN, J.H. y MASÓN, R.O. (2004). "The value of happiness in the workplace", Organizational Dynamics, Vol. 33, n. 4, pp. 379-392.

GENERALITAT VALENCIANA, CONSELLERIA DE BIENESTAR SOCIAL (2010). "Guía de conciliación de la vida personal, familiar y laboral: consideraciones generales. Orientación para su implantación en las empresas".

GOFFEE, R. y JONES, G. (1996). "What holds the modern company together?", Harvard Business Review, NovemberDecember, pp. 133-148.

INSTITUTO ANDALUZ DE LA MUJER (2009). "Guía sobre conciliación de la vida laboral, familiar y personal". Sevilla.

INSTITUTO DE LA MUJER (2008). "De la conciliación a la corresponsabilidad: buenas prácticas y recomendaciones". Madrid.

ISEN, A.M.; DAUBMAN, K.A. y NOWICKI, G.P. (1987). "Positive affect facilitates creative problem solving", Journal of Personality and Social Psychology, Vol. 52, n. 6, pp. 1122-1131.

KARAKAS, F. (2009). "New paradigms in organization development: positivity, spirituality, and complexity", Organization Development Journal, Vol. 27, n. 1, pp. 11-26.

LEE, J. y MILLER, D. (1999). "People matter: commitment to 
employees, strategy and performance in Korean firm", Strategic Management Journal, vol. 20, $\mathrm{n}^{\circ}$ 6, pp. 579-593.

LLANO, A. (1997). "Organizaciones Inteligentes en la sociedad del conocimiento", Cuadernos de Empresa y Humanismo, $\mathrm{n}$. 61.

LÓPEZ LÓPEZ, M.T. y VALIÑO CASTRO, A., "Conciliación familiar y laboral en la Unión Europea: valoración de las políticas públicas". Madrid. Consejo Económico y Social.

LUTHANS, F. y YOUSSEF, C. (2007). "Emerging positive organizational behavior", Journal of Management, vol. $33, n^{\circ} 3$, pp. 321-349.

LYTTLE, J. (2007). "The judicious use and management of humor in the workplace", Business Horizons, Vol. 50, pp. 239-245.

LYUBOMIRSKY, S.; KING, L. y DIENER, E. (2005). "The benefits of frequent positive affect: does happiness lead to success?", Psychological Bulletin, Vol. 131, n. 6, pp. 803-855.

McGREGOR, D. (2006). "The human side of enterprise", McGrawHill.

MALONE, P.B. (1980). "Humor: a double-edged tool for today's managers", Academy of Management Review, July, 5, pp. 357-360.

MARTÍNEZ, MARTÍNEZ, M.C. (2009). "Género y conciliación de la vida familiar y laboral, un análisis psicosocial". Murcia. Universidad de Murcia.

MARKS, N. (2006). "Happiness is a serious business", Reflections on employee engagement, pp. 5-7.

MEDINA ÁVILA, L. (2004). "La paradoja de la felicidad", Revista OIKOS: revista de la Escuela de Administración y Economía, n. 18.

MELÉ CARNÉ, D. (2004). "Conciliar trabajo y familia: un reto para el siglo XXI". Pamplona. Eunsa.

MENDIETA ENRÍQUEZ, M.J. (2005). "Gestión estratégica de personal", Nuevas Tendencias, n. 59, pp. 24-28.

MILNE, J. (2007). "Passion power", Training and Coaching Today, September, p. 29.

MOCCIA, S. y TRIGO, T. (2009). "Trabajar con buen humor en la empresa y siempre". Pamplona. Eunsa.

MYERS, D.G. (2000). "The funds, friends, and faith of happy people", American Psychologist, vol. 55, n 1, 56-67. 
OLCESE, A.; RODRÍGUEZ M.A. y ALFARO, J. (2008). "Manual de la empresa responsable y sostenible". Madrid. McGrawhill.

PAGE, N. et al. (2008). "Gestión positiva de los recursos humanos: aplicaciones de la Psicología Positiva a lo largo del ciclo vital del trabajador" en Vázquez, C., Herves, G., "Psicología Positiva Aplicada". Bilbao. Ed. Desclée de Brower.

QUINTANILLA, I. (2008). "Psicología del marketing social". Valencia. Promolibro.

PETERSON, C. (2000). "The future of optimism", American Psychologist, Vol. 55, n. 1, pp. 44-55.

PETERSON, C.; PARK, N. y SELIGMAN, M.E. (2005). "Orientation to happiness and life satisfaction: the full life versus the empty life", Journal of Happiness Studies, n. 6, pp. 25-41.

PLOYHART, R.E. (2006). "Staffing in the $21^{\text {st }}$ Century: New challenges and strategic opportunities", Journal of Management, Vol. 32, pp. 868-897.

PRIETO CAMPÀ, H. (2006). "Antagonismos entre la vida laboral y la vida personal: Indagación sobre algunas prácticas de conciliación en España, Francia y Portugal". Madrid. Hacer.

REMOR, E. (2008). "Contribuciones de la Psicología Positiva al ámbito de la Psicología de la Salud y Medicina Conductual", en "Psicología Positiva Aplicada". Bilbao. Desclée de Brouwer.

RIVAS, A.M. (2008). "Mujeres y hombres en conflicto: trabajo, familia y desigualdades de género". Madrid. HOAC.

RODRÍGUEZ-PENELAS, H. (2008). "La cultura es también una responsabilidad social empresarial", Revista Empresa y Humanismo, Vol. XI, n. 2, pp. 109-134.

ROMERO, E. y PESCOSOLIDO, A. (2008). "Humor and group effectiveness", Human Relations, Vol. 61, n. 3, pp. 395-418.

ROMERO, E.J. y CRUTHIRDS, K.W. (2006). "The use of humor in the workplace", Academy of Management Perspective, Vol. 20, pp. 58-69.

SALA-i-MARTIN, J. (2009). "El crecimiento económico", Reverté.

SALANOVA SORIA, M. (2008). "Organizaciones saludables y desarrollo de recursos humanos", Revista de trabajo y Seguridad Social, Vol. 47, n. 303, pp. 179-214. 
SCHNEIDER, B. (1987). "The people make the place", Personnel Psychology, Vol. 40, pp. 437-453.

SEO, M., BARRETT, L., BARTUNEK, J. (2004). "The role of affective experience in work motivation", Academy of Management Review, vol. 29, $\mathrm{n}^{\circ}$ 3, pp. 423-439.

SELIGMAN, M., E., P., CSIKSZENTMIHALYI, M. (2000). "Positive Psychology. An introduction", American Psychologist, Vol. 55, n. 1, pp. 5-14.

SELIGMAN, M.E.P.; STEEN, T.A.; PARK, N. y PETERSON, C. (2005). "Positive Psychology Progress. Empirical Validation of interventions", American Psychologist, Vol. 60, n. 5, pp. 410-421.

SHELDON, K.M. y LYUBOMIRSKY, S. (2006). "Achieving sustainable gains in happiness: change your actions not your circumstances", Journal of Happiness Studies, Vol. 86, pp. 55-86.

STAW, B.; SUTTON, R. y PELLED, L. (1994). "Employee positive emotions and favourable outcomes at the workplace", Organization Science, Vol. 51, n. 1, pp. 51-70.

TEEHAN, R. (2008). "Emotional Intelligence, sense of Humor, and Job Satisfaction. Relationship, Affect, and Implication". Alemania. Saarbrücken. VDM Verlag Dr. Müller.

TKACH, C. y LYUBOMIRSKY, S. (2006). "How do people pursue happiness?: relating personality, happiness-increasing strategies and well-being", Journal of Happiness Studies, Vol. 7, pp. 183-225.

TRUSS, K. et al. (2006). "Working Life: Employee Attitudes and Engagement 2006". London. CIPD.

WRIGHT, T.A. y CROPANZANO, R. (2004). "The role of psychological well-being in job performance: a fresh look at an ageold quest", Organizational Dynamics, Vol. 33-4, pp. 338-351.

YIP, J.A. y MARTÍN, R.A. (2006). "Sense of humor, emotional intelligence, and social competence", Journal of Research in Personality, Vol. 40, n. 6, pp. 1202-1208.

ZAMAGNI, S. (2008). "¿Es posible compaginar la competitividad de la empresa con la felicidad?, en "La Ética en las estrategias empresariales del Siglo XXI". Valencia. Fundación ETNOR.

ZELENSKI, J.; MURPHY, S. y JENKINS, D. (2008). "The happyproductive worker thesis revisited", Journal of Happiness Studies, Vol. 9, pp. 521-537. 